\title{
DataVision: A computer-based system for generating and reading digital information accompanying video images on videotape
}

\author{
DENIZ ERGENER and A. RODNEY WELLENS \\ University of Miami, Coral Gables, Florida
}

\begin{abstract}
An Apple Ile-based system is described that generates both machine-readable and humanreadable numbers for recording with visual information on videotape. Hardware and software components of the encoding system are detailed. An automatic decoding system that reads the numbers from videotape into computer memory is also described. Current applications of the system are briefly discussed.
\end{abstract}

Barnes, Haith, \& Roberts (1988) recently described the desirability of merging digital information with video images when recording behavioral events on videotape. The ability to record visual aspects of complex behaviors simultaneously along with digital representations of stimuli or response conditions allows the synchrony of all events to be retained in the permanent visual record. This eliminates the need of creating and managing separate timestamped computer files that may exceed computer memory for lengthy experimental sessions. Having information recorded in computer-readable form eliminates much of the tedium associated with manually decoding events upon videotape playback. Barnes et al. described a system that allows the recording and decoding of a limited amount of digital information on a single line of video that lies above the normally visible video field. The technique requires the construction of customized circuitry for both the encoding and the decoding portions of the system.

The present report describes an alternative system that utilizes off-the-shelf computer and video hardware to generate both machine-readable and human-readable information that occupies a small portion of the visible video field. The display software can generate up to 40 digits of information that occupy 14 lines of a standard 251-line video frame. Upon playback, the user can either read the digits manually or use a customized decoder to read the digitized information directly into a computer.

\section{ENCODING SYSTEM}

The hardware portion of the encoding system consists of an Apple Ile computer and a video mixer. The

This project was supported by funds made available by the Department of Psychology and the College of Arts and Sciences, University of Miami, Coral Gables, FL. The first author is now at AT\&T Bell Laboratories, Middletown, NJ. The manuscript was prepared while the second author was a visiting scientist at the Armstrong Aerospace Medical Research Laboratory, Human Engineering Division, Wright-Patterson Air Force Base, OH. Correspondence may be sent to A. R. Wellens, Department of Psychology, University of Miami, Coral Gables, FL 33124.
Apple IIe computer is equipped with a Video Associates VB-3e Micro-Keyer board. The VB-3e converts the Apple's video output to an NTSC-standard signal that can be genlocked to any other NTSC video source. The converted video signal is combined with a second video source (such as the output from a color television camera; e.g., Panasonic WV-3260) via a commercial video mixer or a special-effects generator (e.g., JVC KM1200SPG). The combined video is then recorded using a high-quality industrial videocassette recorder (VCR; e.g., Panasonic AG-6300). All of these components, including the highquality video equipment, can be purchased "off the shelf' and can be configured with a minimum of effort for about $\$ 7,000$.

The software portion of the encoding system makes use of the high-resolution graphics screen of the Apple Ile computer. Each line of the Apple's graphics screen can be divided into 280 on/off dots. By combining several lines of video, one can create a highly readable set of characters as well as a very reliable digital code.

Figure 1 shows the character set used in the current encoding scheme. Each character fits within a $7 \times 14$ matrix. The upper six lines of each matrix are reserved for a simple digital code consisting of line segments that are seven dots long and are either entirely "on" (white) or entirely "off" (black). This part of the matrix constitutes the machine-readable portion of each character. It is separated from the bottom portion of the matrix by a single blank line. The bottom seven lines are reserved for a more complicated pattern of on/off dots that constitutes the human-readable portion of the characters. As can be seen, there are 10 "visible" characters representing the numerals 0 through 9, plus 6 "invisible" characters that can be used as start or stop characters or as any other kind of character defined by the user.

The Appendix contains a machine-language subroutine entitled "Numbers" that is used to generate the above character set and to write these characters to preselected positions along the top of the high-resolution screen. A 40-position character buffer is reserved in memory for informing the subroutine which of the 16 available charac- 


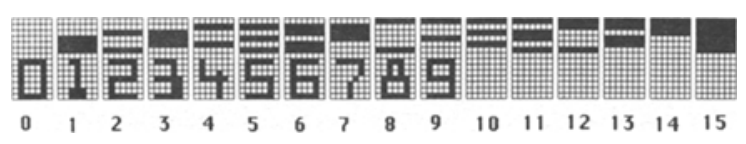

Figure 1. Dot-matrix pattern of the character set generated by the assembly-language program "Numbers."

ters should be placed in each of the 40 high-resolution screen positions.

The Appendix also contains a sample Basic program that can be used to "poke" values into the character buffer and to call the machine-language subroutine to display the buffer. To run the program, an Applied Engineering Time Master II clock card should be installed in Slot 5 in the Apple. Two game paddles or joysticks with pushbuttons should also be connected to the Apple's game port via an SCRG Paddle-Adapple or equivalent game-port expander.

The sample program first interrogates the user to provide a six-digit subject-identification code that will be displayed in Positions 15 through 20 on the high-resolution screen. The user is also asked if the automatic decoder will be used for decoding the information on playback. The program then reads the clock card and pokes the current date and time in Positions 1 through 4 and Positions 7 through 12 , respectively. If the automatic decoder will be used, the date information normally displayed in Positions 1 through 4 is replaced with a four-digit decoder start code. The program then "peeks" to four locations in the Apple Ile's memory reserved as flags for indicating the status of the four game paddle or joystick pushbuttons. It then pokes these values into Locations 37 through $\mathbf{4 0}$ in the display buffer. Finally, the program displays the contents of the buffer on the high-resolution screen. The program continues to update the displayed time and pushbutton information at the rate of three times per sec. Faster update rates are possible using all machinelanguage programming. The update rate depends on the amount of information to be displayed and the amount of preprocessing needed to acquire it. The theoretical limit on display rate is set by the video-frame rate of 30 times per second.

Figure 2 shows the output of a slightly more sophisticated program that uses the Numbers subroutine combined with a split-screen display of 2 subjects involved in a cooperative video game (see Wellens \& Ergener, 1988). The automatic decoding option has been chosen; therefore, Positions 1-4 contain a special start code consisting of three "invisible" characters plus the number " 5 ." This is followed by the date (MTH:DA) in Positions 5-8, the time (HR:MIN) in Positions 10-13, and the assigned subject-identification number in Positions 15-20. The heart rates of up to 4 subjects (each divided by two to fit into a two-digit display format) are displayed in Positions $22-29$, respectively. The state of four momentarycontact switches ( $0=$ off, $1=$ on) used for controlling communication between team members is displayed in
Positions 31-34. Finally, the output of four speechdetection circuits $(0=$ silent, $1=$ talking) used to monitor the vocal activity of up to 4 subjects is displayed in Positions 36-39. As can be seen, there is ample room below the digital information to capture full-face images of 2 subjects as well as the electronic game boards to which they are attending.

\section{DECODING SYSTEM}

When a videotape containing the encoded information is played back on a VCR, the numerals representing the encoded data are clearly visible along the top of the playback monitor. The observer can watch the encoded information change in relation to the behaviors recorded on the remainder of the video image. If the appropriate start code has been included in the display, a specialpurpose decoder can be used to send the encoded information directly to computer memory.

The decoding hardware consists of a special-purpose video-detection circuit connected to an Apple Ile computer via a slot-mounted interface card. The decoding circuit synchronizes itself to the video output of the playback VCR by way of a sync separator that detects the vertical and horizontal sync pulses in the VCR's composite video output. It is important that a high-quality VCR be used for both recording and playback to ensure stable video and synchronizing signals. Decoding of characters within the video field is accomplished via template matching.

A block diagram of the video decoding system is shown in Figure 3. A more detailed schematic diagram showing individual components of the system may be obtained from the authors at no charge. The main components of the system are a sync separator, a control circuit, a video digitizer, and a decoder circuit consisting of a series of shift registers, look-up tables, adders, and a comparator. Raw data are extracted from the VCR composite video signal and processed data are memory-mapped into the Apple Ile via a card plugged into one of its available expansion slots. "Handshaking" occurs between the decoder's control circuit and a machine-language program running as a background program within the Apple Пle.

The decoding system runs as follows. When the sync separator detects a vertical sync pulse that marks the beginning of a new video field, it enables a timing circuit that delays the onset of video sampling until the first line of encoded digital information. At the end of this fixed time period, the digitizer begins sampling each horizontal video line at a rate of 328 samples per line, and continues for six lines. This sampling rate allows some room for timing errors and ensures that the 280 bits of encoded information (40 characters $\times 7$ bits) per line is captured along with some empty bits both preceding and following the critical character set.

The digitizer is a simple comparator circuit the output of which is high if the sampled video signal is at least $60 \%$ over the black level relative to the white level. This allows the digitizer to discriminate between the white and 

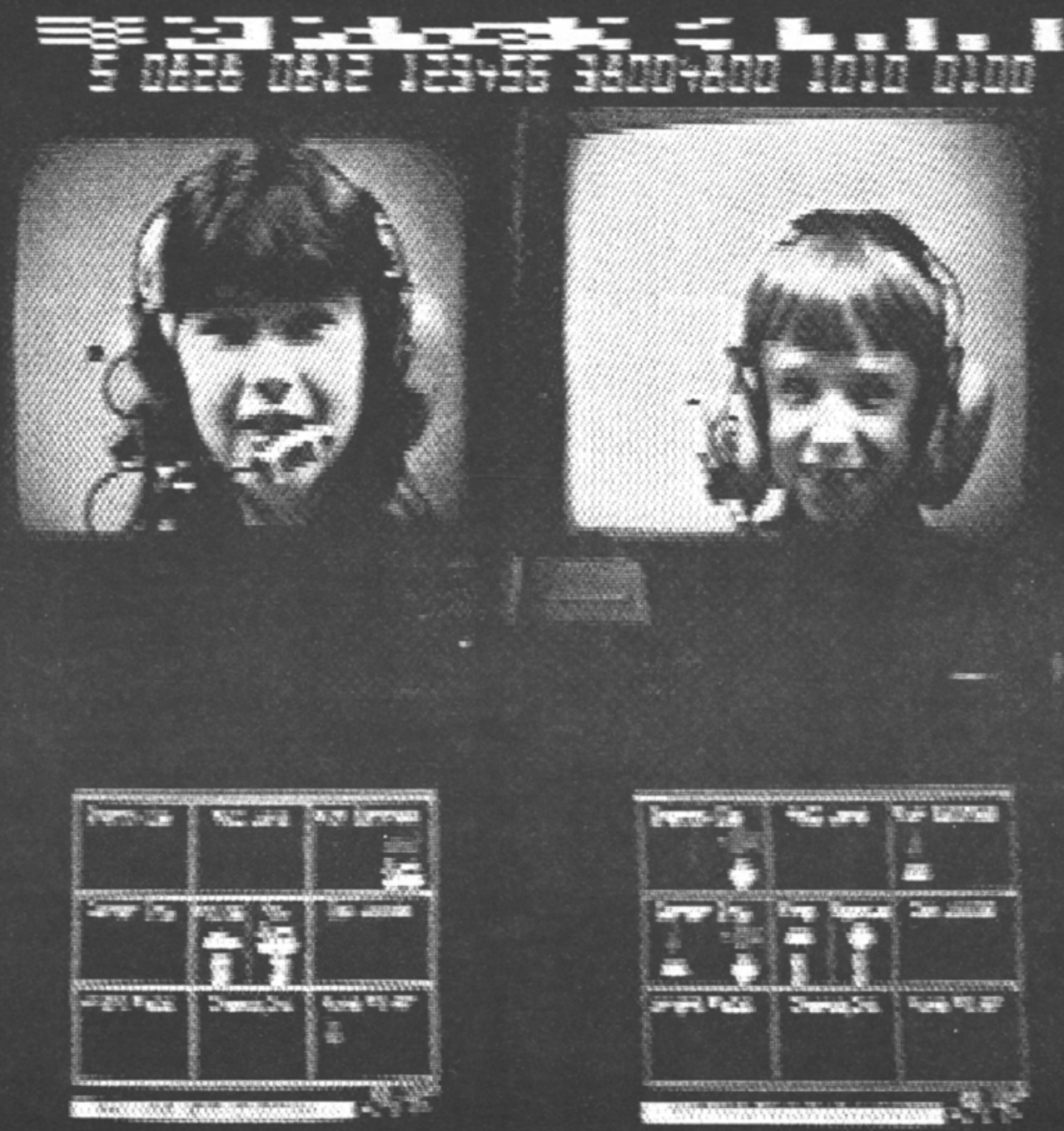

Figure 2. Photo of a single frame of videotape showing the output of "Numbers" inserted at the top of a split-screen image of 2 subjects playing a cooperative computer game.

black dots placed in the video field by way of the encoding system. The output of the digitizer is fed directly into six shift registers, each 328 bits long and chained serially to one another. Thus, when digitizing begins, the bits streaming out of the digitizer wind their way down the series of six shift registers in snake-like fashion. By the end of the digitizing phase, the encoded information from the first line of code for all 40 character positions is contained in the shift register at the bottom of the stack, and the bits for the sixth line of code for all character positions are contained in the shift register at the top of the stack. All data are shifted one bit at a time through the shift registers until the first encoded start character is detected via template matching.
Template matching is accomplished by connecting the last seven bits of each shift register to the first seven address lines of six $2 \mathrm{~K} \times 4$ bit programmable read-only memory (PROM) chips. These PROMs are used as correlators to identify the pattern on each line for each character. Each PROM contains a look-up table for the pattern associated with a particular line of each of the 16 characters. The upper four bits of each PROM, which can be set from the computer interface, are used for selecting one of the 16-character templates.

Correlation in this case is defined as an "exclusive or." If the bits in each location in the template match the incoming data, the result is 1 ; otherwise, it is 0 . The total result for each template comparison is computed by means 


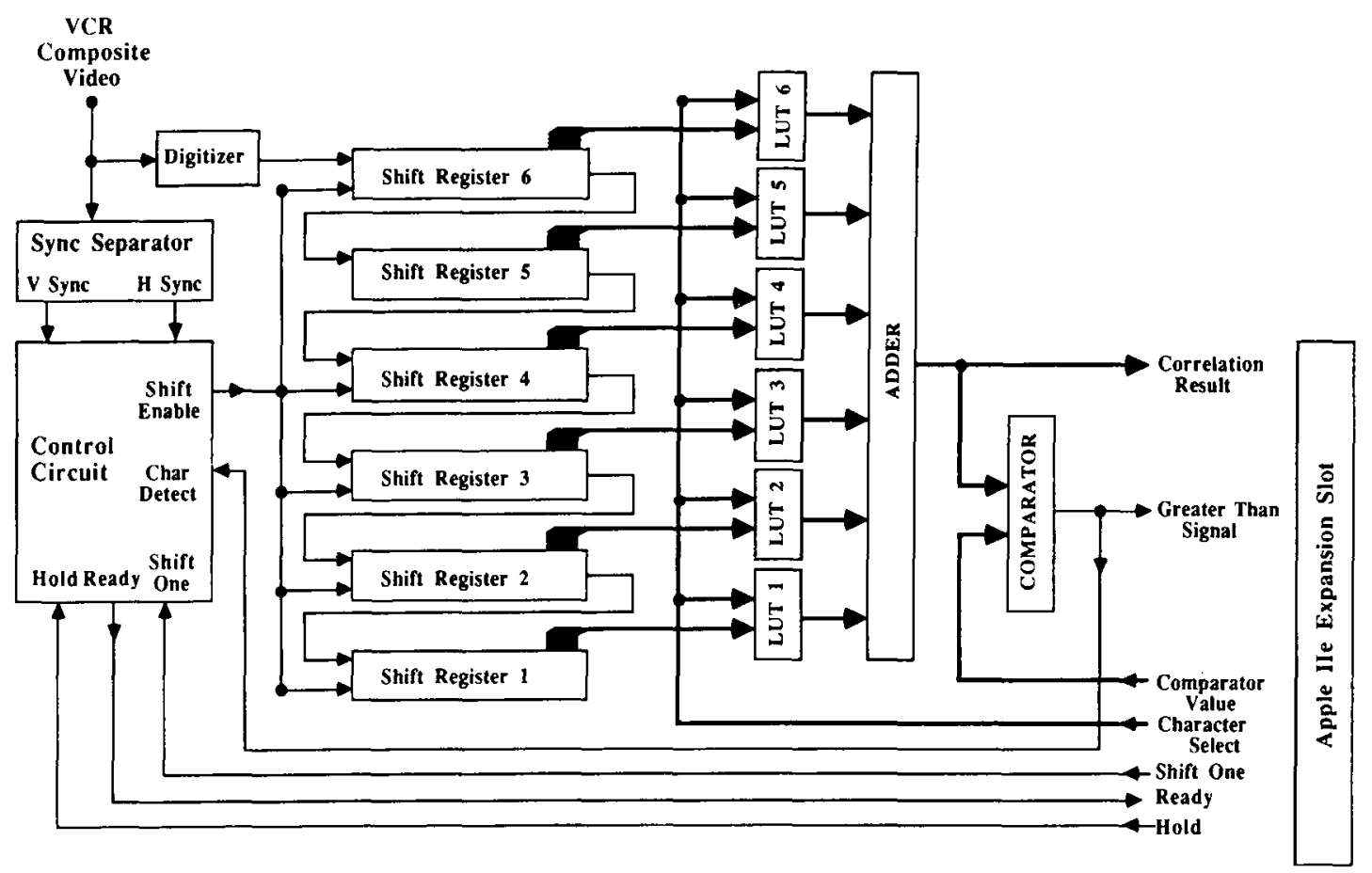

Figure 3. Block diagram of the video decoding system.

of a series of full adders. The maximum value across all six look-up tables is equal to 42 . Any desired threshold value may be placed in a comparator to provide a "character-detect" signal. The total result of the correlator, as well as the result of the comparator match, is available at the computer interface.

The protocol for decoding is as follows. When the computer is ready to receive data, it polls the ready line. The ready line is set during the vertical blanking interval of the incoming video signal when all the shift registers are cleared. The computer then sets the template to the first of four start characters through the upper four bits of the PROMs. The start characters consist of two of the first "invisible characters" shown in Figure 1, followed by the character " 5 ," which is the invisible character's inverse, and then a repeat of the first invisible character.

After the template and threshold values have been set for the first start character, the data in the shift registers are shifted one step at a time until a character-detect signal is sent from the comparator. Receipt of the characterdetect signal automatically initiates a "hold" signal that locks the data in position within the shift registers. Then a check for a false detect is accomplished by changing the template to the " 5 " character and initiating shifting once again. If the comparator detects the " 5 " character within an acceptable number of shifts, the template is assumed to be synchronized with the character positions, and the rest of the data is analyzed. Otherwise, there is a false detect and the computer disables the hold and waits for the next ready signal.

Once the start characters have been confirmed, the retrieval of data is accomplished by sending seven shift signals at a time to the control circuit, and comparing the contents of the last seven bits of each shift register with each template and selecting the maximum correlation obtained as the character detected in that position. This process is repeated for the remainder of the 40 data positions. When all the data have been sent to the computer, the hold is disabled and the process is repeated for the next frame of video. This method of template matching is extremely quick and can be accomplished in real time.

\section{CURRENT APPLICATIONS}

The DataVision system has been used primarily to record and decode physiological information in combination with videotaped records of behavioral events. An early version of the system that "hid" encoded information above the normally visible video field, similar to that reported by Barnes et al. (1988), was used by Cardon (1985) to record beat-to-beat heart rate information of subjects who were being interviewed over a two-way television system (Wellens, 1979). Experience with this early system led to the development of the current "visible" system that records highly readable characters within the video "safe zone" and allows the experimenter to monitor the status of all recorded information continually.

More recent studies have also used the DataVision system. Pozo, Carver, Wellens, and Scheir (1988) used the system to record heart-rate and blood-pressure information of subjects while simultaneously recording closeup facial records of each subject and a pretaped interviewer (see Wellens \& Ergener, 1986). Gonzalez et al. (in press) used the system to record the heart rate of children, along 
with their behavioral reactions, while receiving hypodermic injections. Wellens (1987) used the system, as configured in Figure 2, to record heart rate and talking data of team members playing a cooperative computer game. A duplicate of the last system is currently being used in a series of experiments dealing with team performance at the Armstrong Aerospace Medical Research Laboratory at Wright-Patterson Air Force Base.

Because the encoding portion of the DataVision system requires no customized circuitry, several experimenters can collect data independently using their own Apple Ile computers and VCRs. As videotapes accumulate, a single decoder can be used as a central resource. For some applications, when experimenters simply want to keep a permanent record of running time and date information on their videotapes, no automatic decoder is needed.

\section{AVAILABILITY}

The Appendix contains the machine-language software needed to generate the character set used in the DataVision system. The Appendix also contains a sample Basic program that demonstrates how to poke data to a memory buffer for display on the Apple's high-resolution graphics screen. This software, together with the computer and video hardware described in the preceding text, is all that is needed for the encoding part of the system. The decoding part of the system is more complex and requires several customized chips and an experienced engineer for constructing and testing the digital circuits. Schematics for the decoding circuit and its accompanying control circuit are available free of charge from the authors. Alternative methods of decoding that may simplify the decoding process are currently being explored. Interested researchers should contact the authors for the latest developments.

\section{REFERENCES}

Barnes, O., Haith, M. M., \& Roberts, R. J., Jr. (1988). Simultaneous electronic recording of video and digital information on the video channel of a VTR or VCR. Behavior Research Methods, Instruments, \& Computers, 20, 32-36.

CARDON, M. C. (1985). Effects of shifts in interview topic intimacy upon interviewee looking behavior. Unpublished master's thesis, University of Miami, Coral Gables, FL.

Gonzalez, J. C., Routh, D. K., SaAb, P. G., Armstrong, F. D., Schifman, L., Guerra, E., \& Fawcett, N. (in press). Effects of parent presence on children's reactions to injection: Behavioral, physiological and subjective aspects. Joumal of Pediatric Psychology.

Pozo, C., Carver, C. S., Wellens, A. R., \& Scheir, M. S. (1988). Judging facial feedback of others' reactions to oneself: The biasing effect of social anxiety. Manuscript in preparation.

WELlens, A. R. (1979). An interactive television laboratory for the study of social interaction. Journal of Nonverbal Behavior, 7, 121-123.

WELLENS, A. R. (1987). Effects of telecommunication media upon group decision making processes within a multi-team situation assessment task. (Final Report, Contract No. F49620-85-C-0013/SB5851-0360, PO S-760-6MG-085). Bolling Air Force Base, DC: Air Force Office of Scientific Services.

WELLENS, A. R., \& ERGENER, D. (1986). An improved video multiplexer for behavioral research. Behavior Research Methods, Instruments, \& Computers, 18, 480-481

Wellens, A. R., \& ERGener, D. (1988). The C.I.T.I.E.S. game: A computer-based situation assessment task for studying distributed decision making. Simulation \& Games, 19, 304-327.

\section{APPENDIX}

THE FOUOWING IS A SOURCE CODE USTING FOR AN ASSEMBLY LANGUAGE SUBROUTINE THAT WRITES A CUSTOM DESIGNED CHARACTER SET TO THE UPPER 14 LNES OF THE APPLE Ile HIGH RESOLUTION SCREEN. THE SUBROUTINE USES TWO MACROS, "WRITE" AND "DISP" AND CAN BE ASSEMBLED USING THE MERLIN "PRO" 6502 MACRO ASSEMBLER. ONCE ASSEMBLED AND LOADED AT LOCATION A\$800, THE CONTENTS OF A 40 POSITION BUFFER, LOCATED AT LOCATIONS 2522 - 2561, WILL BE DISPLAYED WHEN THE SUBROUTINE IS CALED AT 2048. THE CONTENT OF THE DISPLAY BUFFER CAN BE CHANGED BY POKING THE DESIRED CHARACTER VALUES TO INDIVIDUAL BUFFER LOCATIONS.

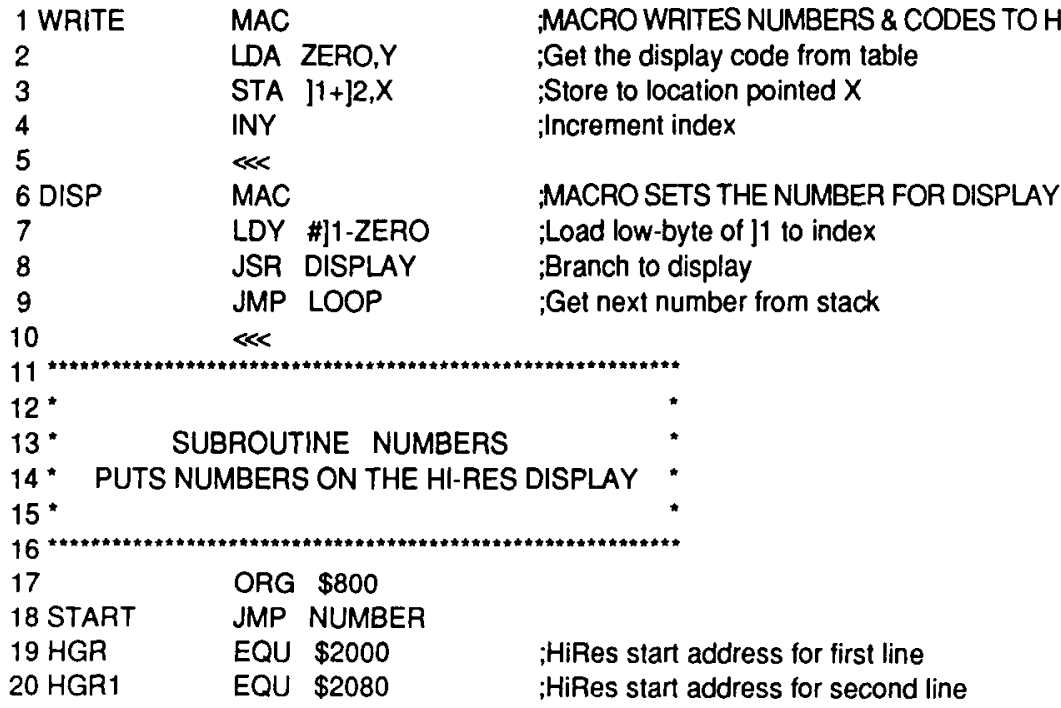


APPENDIX (Continued)

\begin{tabular}{|c|c|c|c|}
\hline 21 NUMBER & LDX & $\#-1$ & ;RESET CHAR COUNTER \\
\hline 22 LOOP & INX & & ;increment counter \\
\hline 23 & $\mathrm{CPX}$ & $\# \$ 28$ & ;Check for end \\
\hline 24 & BEQ & :LAST & ;if yes golo end \\
\hline 25 & LAA & STACK,X & ;Otherwise load next char \\
\hline 26 & AND & $\# \$ O F$ & ;Mask out high bits \\
\hline 27 & ASL & A & ;Multiply by 2 \\
\hline 28 & STA & +4 & ;Set jump address for indrect jump \\
\hline 29 & JMP & (JTABLE) & ; Jump to display routine for char \\
\hline $30:$ LAST & RTS & & ;Return \\
\hline 31 DISPO & DISP & ZERO & \\
\hline 32 DISP1 & DISP & ONE & \\
\hline 33 DISP2 & DISP & TWO & \\
\hline 34 DISP3 & DISP & THREE & \\
\hline 35 DISP4 & DISP & FOUR & \\
\hline 36 DISP5 & DISP & FIVE & \\
\hline 37 DISP6 & DISP & SIX & \\
\hline 38 DISP7 & DISP & SEVEN & \\
\hline 39 DISP8 & DISP & EIGHT & \\
\hline 40 DISP9 & DISP & NINE & \\
\hline 41 DISPA & DISP & AAAA & \\
\hline 42 DISPB & DISP & BBBB & \\
\hline 43 DISPC & DSPP & $\operatorname{cocc}$ & \\
\hline 44 DISPD & DISP & DDDD & \\
\hline 45 DISPE & DISP & EEEE & \\
\hline $\begin{array}{l}46 \text { DISPF } \\
47 \text { DISPLAY }\end{array}$ & $\begin{array}{l}\text { DISP } \\
\text { WRIT }\end{array}$ & $\begin{array}{l}\text { FFFF } \\
\text { IE HGR;0 }\end{array}$ & \\
\hline 48 & WRIT & TE HGR;1024 & \\
\hline 49 & WRIT & TE HGR;2048 & \\
\hline 50 & WRIT & IE HGR;3072 & \\
\hline 51 & WRIT & TE HGR;4096 & \\
\hline 52 & WRIT & TE HGR;5120 & \\
\hline 53 & WRIT & TE HGR;6144 & \\
\hline 54 & WRIT & TE HGR;7168 & \\
\hline 55 & WRIT & TE HGR $1 ; 0$ & \\
\hline 56 & WRIT & TE HGR1;1024 & \\
\hline 57 & WRIT & TE HGR1;2048 & \\
\hline 58 & WRIT & TE HGR1;3072 & \\
\hline 59 & WRIT & TE HGR1;4096 & \\
\hline 60 & WRIT & TE HGR1;5120 & \\
\hline 61 & RTS & & \\
\hline 62 ZERO & DFB & \\
\hline 63 ONE & DFB & \multicolumn{2}{|c|}{$0,0,0,127,127,127,0,12,8,8,8,8,28,28$} \\
\hline 64 TWO & DFB & \multicolumn{2}{|c|}{$0,0,127,0,0,127,0,62,32,32,62,2,2,62$} \\
\hline 65 THREE & DFB & \multicolumn{2}{|c|}{$0,0,127,127,127,0,0,30,16,16,62,48,48,62$} \\
\hline 66 FOUR & DFB & \multicolumn{2}{|c|}{$0,127,0,0,127,0,0,2,2,18,62,16,16,16$} \\
\hline 67 FIVE & DFB & \multicolumn{2}{|c|}{$0,127,0,127,0,127,0,62,2,2,62,32,32,62$} \\
\hline $68 \mathrm{SIX}$ & DFB & \multicolumn{2}{|c|}{$0,127,127,0,127,127,0,62,2,2,62,34,34,62$} \\
\hline 69 SEVEN & DFB & \multicolumn{2}{|c|}{$0,127,127,127,0,0,0,62,32,32,16,8,8,8$} \\
\hline $70 \mathrm{EIGHT}$ & DFB & \multicolumn{2}{|c|}{$127,0,0,0,127,127,0,28,20,20,62,34,34,62$} \\
\hline 71 NINE & DFB & \multicolumn{2}{|c|}{$127,0,0,127,0,0,0,62,34,34,62,32,32,62$} \\
\hline 72 AAAA & DFB & \multicolumn{2}{|c|}{$127,0,127,0,127,0,0,0,0,0,0,0,0,0$} \\
\hline 73 BBBB & DFB & \multicolumn{2}{|c|}{$127,0,127,127,0,127,0,0,0,0,0,0,0,0$} \\
\hline $74 \mathrm{CCCC}$ & DFB & \multicolumn{2}{|c|}{$127,127,0,0,0,127,0,0,0,0,0,0,0,0$} \\
\hline 75 DDDD & DFB & \multicolumn{2}{|c|}{$127,127,0,127,127,0,0,0,0,0,0,0,0,0$} \\
\hline 76 EEEE & DFB & \multicolumn{2}{|c|}{$127,127,127,0,0,0,0,0,0,0,0,0,0,0$} \\
\hline 77 FFFF & DFB & $127,127,127,12$ & $127,127,0,0,0,0,0,0,0,0$ \\
\hline 78 STACK & DS & START $+\$ 500-^{*}$ & \\
\hline & & & \\
\hline 80 •|III!!!!I!I! & IIIIII & MAKE SURE TH & T FOLLOWING TABLE \\
\hline $\begin{array}{l}81 \text { "II!!!!!!!!!! } \\
82 \text { * }\end{array}$ & !!!!! IS & AT THE BEGINII & OF A MEMORY PAGE \\
\hline 83 JTABLE & DA & DISPO & Indirect jump address for ZERO \\
\hline 84 & $\mathrm{DA}$ & DISP1 & indirect jump address for ONE \\
\hline 85 & DA & DISP2 & ;Indirect jump address for TWO \\
\hline 86 & DA & DISP3 & ;Indirect jump address for THREE \\
\hline
\end{tabular}


APPENDIX (Continued)

\begin{tabular}{|c|c|c|c|}
\hline 87 & $\mathrm{DA}$ & DISP4 & ;Indirect jump address for FOUR \\
\hline 88 & $\mathrm{DA}$ & DISP5 & ;Indirect jump address for FIVE \\
\hline 89 & $\mathrm{DA}$ & DISP6 & ;Indirect jump address for SIX \\
\hline 90 & $\mathrm{DA}$ & DISP7 & ;Indirect jump address for SEVEN \\
\hline 91 & $\mathrm{DA}$ & DISP8 & ;Indirect jump address for EIGHT \\
\hline 92 & $\mathrm{DA}$ & DISP9 & ;Indirect jump address for NINE \\
\hline 93 & DA & DISPA & ;Indirect jump address for AAAA \\
\hline 94 & DA & DISPB & ;ndirect jump address for BBBB \\
\hline 95 & DA & DISPC & ;Indirect jump address for CCCC \\
\hline 96 & DA & DISPD & ;Indirect jump address for DDDD \\
\hline 97 & $\mathrm{DA}$ & DISPE & ;Indirect jump address for EEEE \\
\hline 98 & DA & DISPF & ;Indirect jump address for FFFF \\
\hline 99 :END & RTS & & \\
\hline
\end{tabular}

THE FOLLOWING BASIC PROGRAMUTIUZES THE ASSEMBLY LANGUAGE SUBROUTINE "NUMBERS" TO DISPLAY A USER SUPPLED SUBJECT ID NUMBER, CURRENT TIME AND DATE INFORMATION, AND THE STATUS OF FOUR PADDLE PUSH-BUTTONS ON THE APPLE II HIGH RESOLUTION SCREEN. IT ASSUMES AN APPLED ENGINEERING TIME MASTER II CLOCK CARD HAS BEEN PLUGGED INTO SLOT 5 AND THAT FOUR GAME PADDLES HAVE BEEN PLUGGED INTO THE GAME PORT VIA A SCRG PADDLE-ADAPPLE VO EXPANSIONADAPTOR.

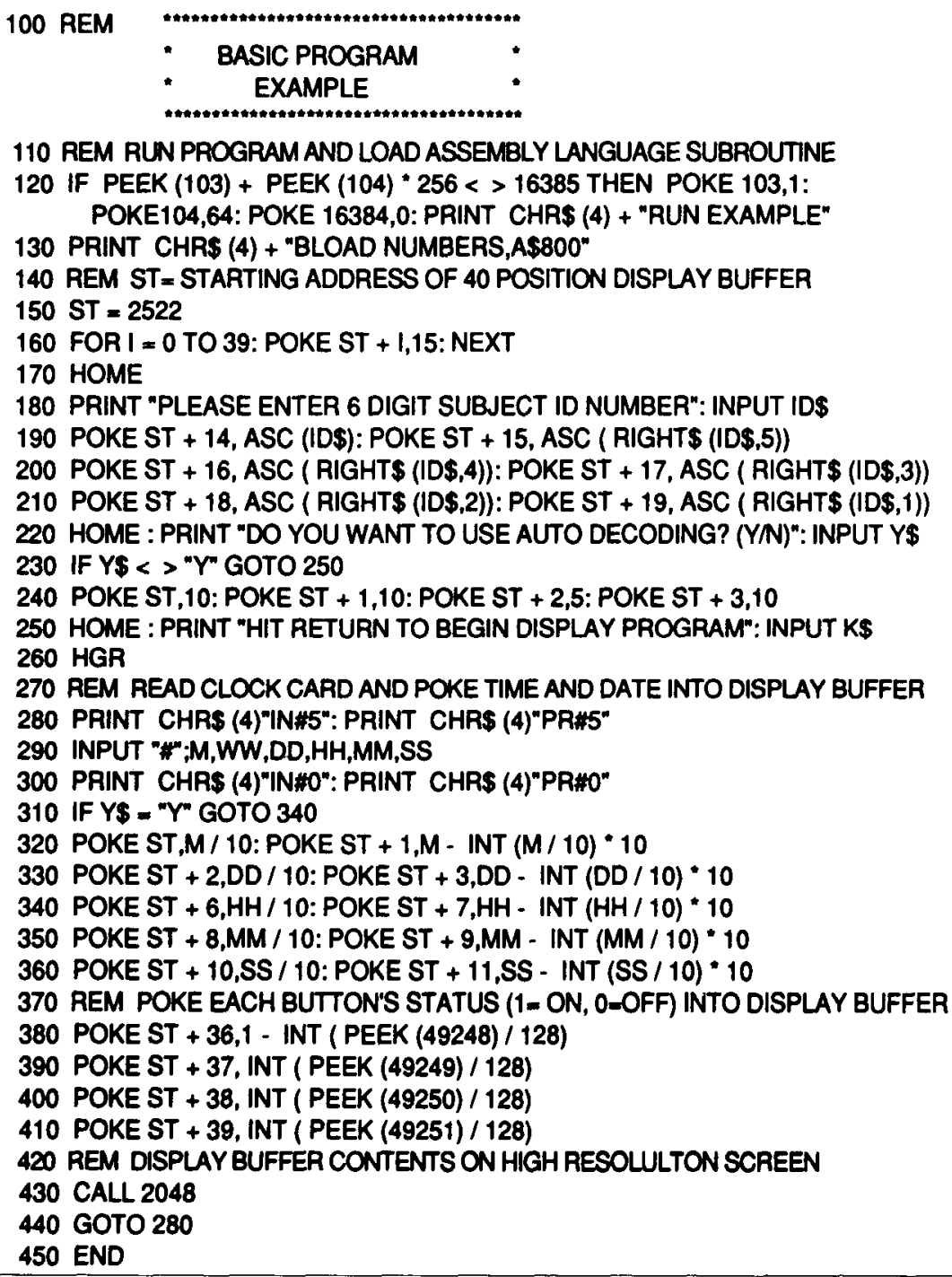

